\title{
Functional impairment of Tax-specific but not CMV-specific CTLs in a minor population of asymptomatic HTLV-1-carriers
}

\author{
Atsuhiko Hasegawa ${ }^{1 *}$, Ayako Takamori ${ }^{1}$, Atae Utsunomiya ${ }^{2}$, Yasuhiro Maeda ${ }^{3}$, Yoshihisa Yamano ${ }^{4}, Y_{\text {Ykiko Shimizu }}^{4}$, \\ Yotaro Tamai ${ }^{1}$, Amane Sasada ${ }^{1}$, Na Zeng ${ }^{1}$, Ilseung Choi ${ }^{5}$, Naokuni Uike ${ }^{5}$, Jun Okamura ${ }^{6}$, Toshiki Watanabe ${ }^{7}$, \\ Takao Masuda ${ }^{1}$, Mari Kannagi ${ }^{1}$
}

From 15th International Conference on Human Retroviruses: HTLV and Related Viruses Leuven and Gembloux, Belgium. 5-8 June 2011

Human T-cell leukemia virus type 1 (HTLV-1) causes adult T-cell leukemia (ATL) and HTLV-I-associated myelopathy/tropical spastic paraparesis (HAM/TSP) in a small percentage of infected individuals. ATL is often associated with general immune suppression and an impaired HTLV-1-specific T cell response. We previously found that a small fraction of asymptomatic HTLV-1-carriers (AC) also showed impaired T cell responses against the major target antigen Tax. However, it is unclear whether the impaired HTLV-1-specific $\mathrm{T}$ cell response in these individuals is an HTLV-1-specific phenomenon, or merely reflects general immune suppression. In this study, we investigated the function of Tax-specific cytotoxic T lymphocytes (CTLs) using tetramers consisting of major CTL epitopes and HLAA0201, A2402, or A1101 in various HTLV-1-infected individuals possessing these HLAs. Tax-specific CTLs were detected in $33.3 \%(n=7 / 21), 100 \%(n=18 / 18)$, and 86.4\% $(\mathrm{n}=19 / 22)$ of chronic ATL (cATL) patients, HAM/TSP patients, and AC, respectively. Tax-specific CTLs of all HAM/TSP patients tested proliferated well in culture and produced IFN- $\gamma$ when stimulated with Tax peptides, while Tax-specific CTLs detected in cATL patients did not. In ACs, the Tax-specific CTL responses were retained in most cases. However, Taxspecific CTLs in one AC hardly produced IFN- $\gamma$ and failed to proliferate or express a degranulation (CD107a) marker upon Tax peptide stimulation. In contrast, cytomegalovirus (CMV) pp65-specific CTLs in the same donor could be fully activated by pp65 peptide stimulation. These findings indicated that HTLV-1-specific T cell function is impaired in a limited population in an HTLV-1-specific manner during an asymptomatic stage.

\section{Author details}

'Department of Immunotherapeutics, Tokyo Medical and Dental University, Tokyo, 113-8519, Japan. ${ }^{2}$ Department of Hematology, Imamura Bun-in Hospital, Kagoshima, Japan. ${ }^{3}$ Division of Hematology, Department of Internal Medicine, Kinki University School of Medicine, Osaka, Japan. ${ }^{4}$ Department of Molecular Medical Science, Institute of Medical Science, St. Marianna University School of Medicine, Kawasaki, Japan. ${ }^{5}$ Department of Hematology, National Kyushu Cancer Center, Fukuoka, Japan. ${ }^{6}$ Institute for Clinical Research, National Kyushu Cancer Center, Fukuoka, Japan. 'Laboratory of Tumor Cell Biology, Department of Medical Genome Science, Graduate School of Frontier Sciences, The University of Tokyo, Tokyo, Japan.

Published: 6 June 2011

doi:10.1186/1742-4690-8-S1-A101

Cite this article as: Hasegawa et al:: Functional impairment of Taxspecific but not CMV-specific CTLs in a minor population of asymptomatic HTLV-1-carriers. Retrovirology 2011 8(Suppl 1):A101.

\footnotetext{
* Correspondence: hase.impt@tmd.ac.jp

'Department of Immunotherapeutics, Tokyo Medical and Dental University,

Tokyo, 113-8519, Japan

Full list of author information is available at the end of the article
}

(c) 2011 Hasegawa et al; licensee BioMed Central Ltd. This is an open access article distributed under the terms of the Creative 